\title{
Ad_Hoc on Demand Distance Vector Routing using MANET
}

\author{
Shadab Ahmed \\ Rajasthan Technical University, Kota
}

\begin{abstract}
Load balancing is a crucial problem in mobile ad hoc networks. Many conventional routing protocols that are developed are not having functionality of coping up load balancing. Hence several kinds of approaches are followed in the design and development of load balancing routing protocols. This paper aims to survey research articles pertaining to load balancing research problem in mobile ad hoc networks. An ad_hoc network is the cooperative engagement of a collection of mobile nodes without the required intervention of any centralized access point or existing infrastructure. In this paper we present Ad_hoc On Demand Distance Vector Routing (AODV), a novel algorithm for the operation of such ad_hoc networks. Each Mobile Host operates as a specialized router and routes are obtained as needed on demand with little or no reliance on periodic advertisements. AODV provides loop free routes even while repairing broken links. Because the protocol does not require global periodic routing advertisements, the demand on the overall bandwidth available to the mobile nodes is substantially less than in those protocols that do necessitate such advertisements.
\end{abstract}

Keywords: Demand Distance Vector Routing (AODV), MANET, peer-to-peer, topology, Mobile Ad Hoc Networking.

\section{INTRODUCTION}

Mobile ad hoc network is a type of wireless network which contains of mobile nodes having the capability to deploy anytime anywhere without or minimum infrastructure. The applications for mobile ad hoc networks are wide open such as disaster management, emergency operations, rescue operations and many more. One of the major application outcomes of mobile ad hoc network is vehicular ad hoc network. Some important characteristics of mobile ad hoc networks are dynamic topology, peer-to-peer fashion during data transfer, mobility of nodes and in real-time such networks are heterogeneous. The nodes that are present in the mobile ad hoc network moves arbitrarily that leads to frequent topology changes. Due to this, data transfer suffers from channel loses and reliable transfer is becoming a challenging task. Hence several routing protocols are developed. The protocols that are designed and developed for mobile ad hoc networks can be classified into three major divisions such as proactive or table-driven, reactive or on-demand.

Ad_hoc On_Demand Distance Vector Routing

Mobile Ad hoc Network (MANET) is a group of mobile wireless nodes that form a network independently of any centralized administration, while forwarding packets to each other in a multi-hop fashion. In Mobile Ad Hoc Networking, the communication does not rely on any existing infrastructure such as dedicated routers, transceiver base stations or even cables. Mobile devices with wireless radio equipment are supposed to

Communicate with each other, without the help of any other (fixed) devices.

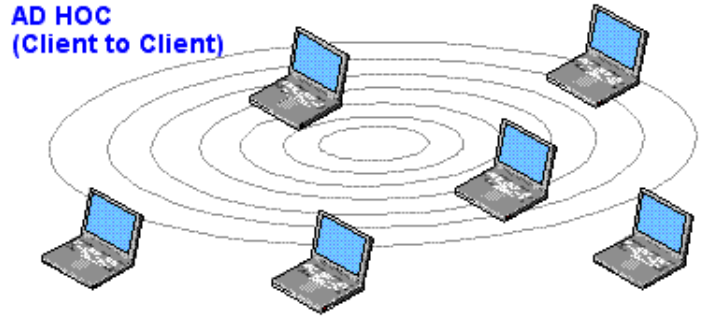

Routing protocols for MANETs can be classified in several ways one of the classifications is according to the number of paths, uni-path routing protocols and multipath routing protocols. In uni-path routing protocols: one route is used to deliver data from source node to destination node while in multipath routing protocols more than one route is used to deliver the data.

In this paper we propose a new route maintenance algorithm to avoid route breaks. Thus, we propose this route maintenance algorithm based on AODV to avoid route breaks.

\section{PROPOSE METHODOLOGY}

\section{AODV Routing Protocol:}

AODV is one of the routing protocols under study by MANET and the typical protocol of on-demand types. In AODV, each node has the routing table, and the freshness of routes is ensured with the sequence number of each the routing information. When each node receives a control packet that occurred in ondemand, the routing table is updated based on the sequence number or the number of 
hops. If a route to a destination is needed, it is established that it has already processed, it discards the RREQ, so the at the route discovery phase and is maintaine at the route loop is avoided and the overhead becomes low. maintenance phase.

\section{Route Discovery:}

When a source node needs a route to a destination node and there is not the valid route in the routing table, the source node broadcasts a route request packet (RREQ) to the destination node.

The RREQ contains the following Felds_

<source_addr, source_sequence, broadcast_id,

dest_addr_,dest_sequence, hop_cnt $>$

When each node receives the RREQ, it creates or updates a reverse route to the source node in the routing table. If it does not have a valid route to the destination node in the routing table, it rebroadcasts the RREQ. When the RREQ flooding from the source node arrives at the destination node, the destination node creates or updates the reverse route. And it unicasts a route reply packet (RREP) which has an incremented the sequence number to the reverse route. When each node receives the RREP, it creates or updates a forward route to the destination node and it forwards the RREP to the reverse route. When the RREP arrives at the source node along with the reverse route, it creates or updates the forward route, and starts communications.

Every node maintains two separate counters:

Sequence number, Broadcast-id (increments whenever the suorce issues a new RREQ)

The source requests using RREQ broadcasting:

<source_addr, source_sequence\#, broadcast_id, dest_addr, dest_sequence\#, hop_cnt> Destination number of RREQ is the last known number to the source

\section{The destination replies using RREP (Route Reply)} unicasting :

<source_addr, dest_addr, dest_sequence\#, hop_cnt, lifetime $>$

The sequence number is first incremented if it is equal to the number in the request RREP contains the current sequence number, hop count $=0$, full lifetime

\section{Intermediate nodes:}

Discard duplicate requests Replies if it has an active route with higher destination sequence number Otherwise broadcasts the request on all interfaces.

For example, Figure 1-I shows the process of the route discovery, which the source node $S$ broadcasts the RREQ and the destination node D unicasts the RREP. If each node has the valid route to the destination node in the routing table when it receives the RREQ, it unicasts the RREP to the source node instead of the destination node. For example, Figure 1-II shows such a process, which the node B unicasts the RREP instead of the node D. During the route discovery, when each node receives the RREQ

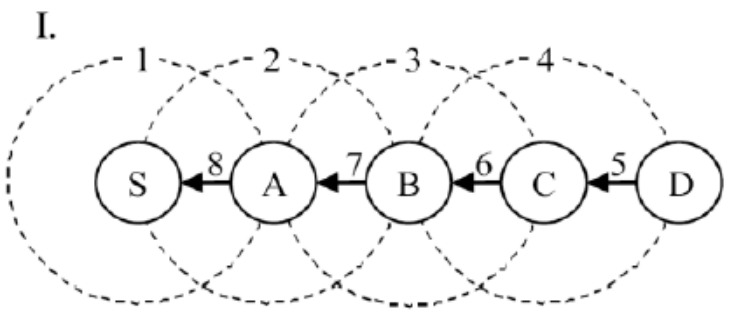

II.

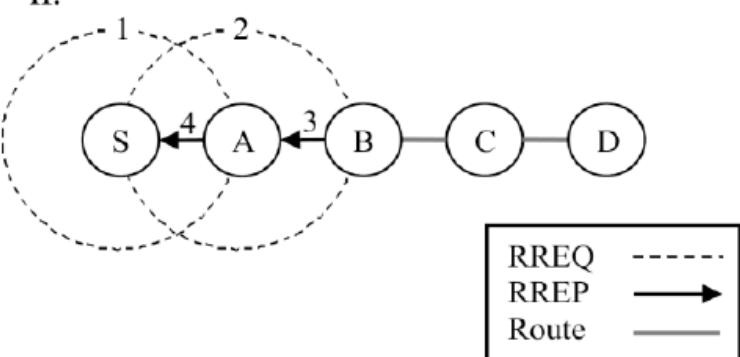

Figure 1. The processes of route discovery

\section{Reverse Path Setup:}

There are two sequence numbers (in addition to the broadcast id)included in a RREQ: the source sequence number and the last destination sequence number known to The source sequence number is used to maintain freshness information about the reverse route to the source, and the destination sequence number specifies how fresh a route to the destination must be before it can be accepted by the source.

As the RREQ travels from a source to various destinations. it automatically sets up the reverse path from all nodes back to the source as illustrated in Figure 2.

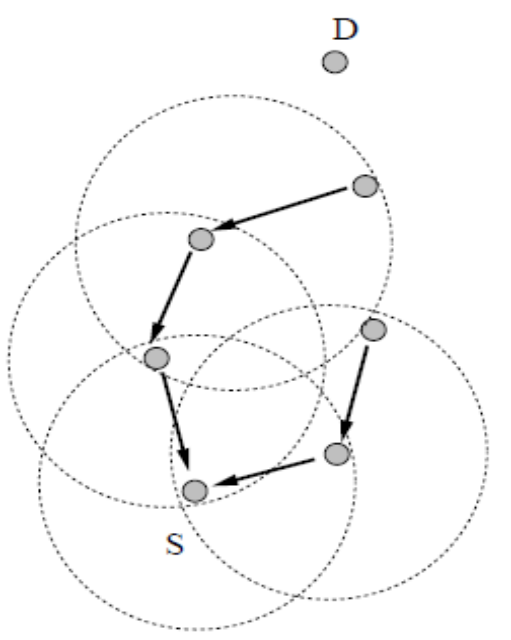

Figure 2. Reverse Path Formation

To set up a reverse path,a node records the address of the neighbor from which it received the first copy of the RREQ: These reverse path route entries are maintained for 
at least enough time for the RREQ to traverse the network and produce a reply to the sender

\section{Forward Path Setup}

Eventually,a RREQ will arrive at a node (possibly the destination itself)that possesses a current route to the destination.The receiving node first checks that the RREQ was received over a bi_directional link, I an intermediate node has a route entry for the desired destination, it determines whether the route is current by comparing the destination sequence number in its own route entry to the destination sequence number in the RREQ. If the RREQ as sequence number for the destination is greater than that recorded by the intermediate node, the intermediate node must not use its recorded route to respond to the RREQ. Instead, the node rebroadcasts the RREQ. The intermediate node can reply only when it has a route with a sequence number that is greater than or equal to that contained in the RREQ.If it does have a current route to the destination, and if the RREQ has not been processed previously, the node then unicasts a route reply packet (RREP)back to its neighbor from which it received the RREQ.

By the time a broadcast packet arrives at a node that can supply a route to the destination, a reverse path has been established to the source of the RREQ .As the RREP travels back to the source,each node along the path sets up a forward pointer to the node from which the RREP came up dates its timeout information for route entries to the source and destination, and records the latest destination sequence number for the requested destination.Figure 3 , represents the forward path setup as the RREP travels from the destination $\mathrm{D}$ to the source node $\mathrm{S}$.Nodes that are not along the path determined by the RREP will timeout after ACTIVE ROUTE TIMEOUT and will delete the reverse pointers.A node receiving an RREP propagates the first RREP for a given source node towards that source.

If it receives further RREPs, it updates its routing information and propagates the RREP only if the RREP contains either a greater destination sequence number than the previous RREP, or the same destination sequence number with a smaller hopcount.

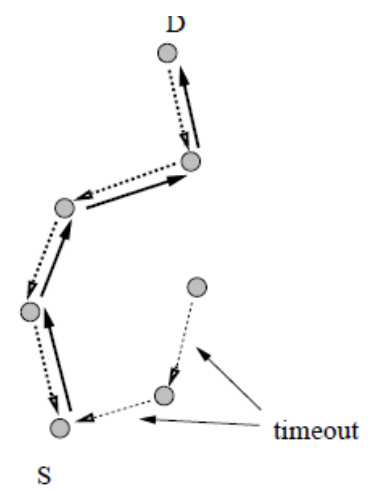

Figure 3. Forwar Path Formation Route Maintenance
It suppresses all other RREPs it receives.This decreases the number of RREPs propagating towards the source while also ensuring the most up to date and quickest routing information. The source node can begin data transmission as soon as the first RREP is received,and can later update its routing information if it learns of a better route.

Each node broadcasts a Hello packet periodically for local connectivity. It broadcasts the RREP with TTL $=1$ as the Hello packet. When the node does not receive any packets from a neighbor during a few seconds, it assumes a link break to the neighbor. In addition, when the node has the link break to the neighbor based on an acknowledgment of MAC layer, it detects a route break to the destination node that the next hop of the route is the neighbor.

When the node that detects the link break is close to the destination node (that is to say the number of hops to the destination node is smaller than the number of hops to the source node), it requires a new route to the destination node, which is known as Local Repair.

The local repair is the route discovery which is similar to the description above. During the local repair, arrival data packets received are buffered. When the RREP is received and the local repair is successful, the node starts sending data packets in the buffer.

For example, Figure 4-I shows the process of the local repair after the link break between the node B and the node $\mathrm{C}$. On the other hand, when the node that detects the link break is far from the destination node, or when the local repair is unsuccessful, the node propagates a route error packet (RERR), which contains the addresses of the unreachable destination, toward the source node.

When each intermediate node receives the RERR, the routes which have the unreachable destination node and have the next hop which is the propagation node of the RERR are made invalid, and it propagates the RERR again. When the source node receives the RERR, the route to the destination node is made invalid similarly and it rediscovers the route again. For example, Figure 2-II shows the process of the route maintenance after the link break between the node A and the node B.

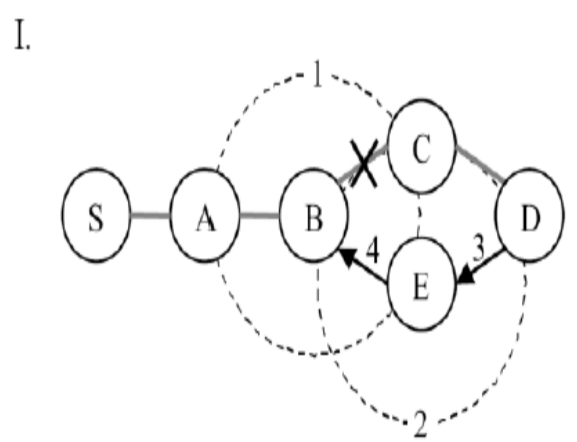




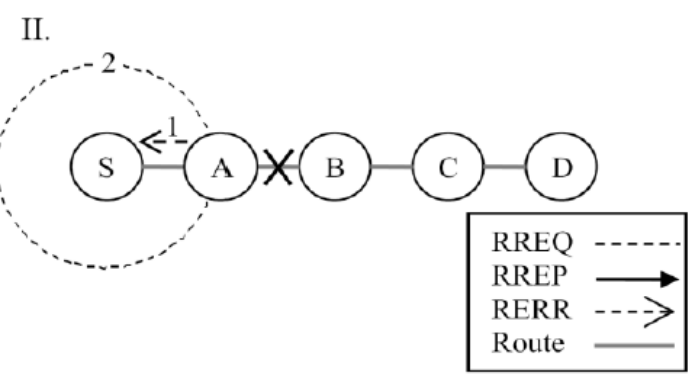

Figure 4. The processes of route maintenance

\section{Algorithm:}

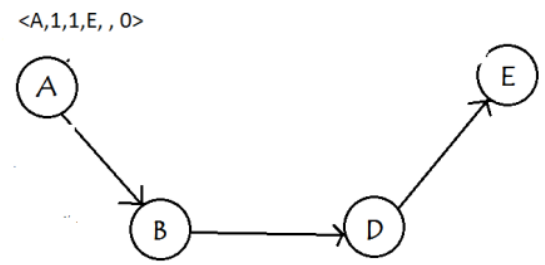

Step 3: Now increase the hop count and broadcast the packet again.

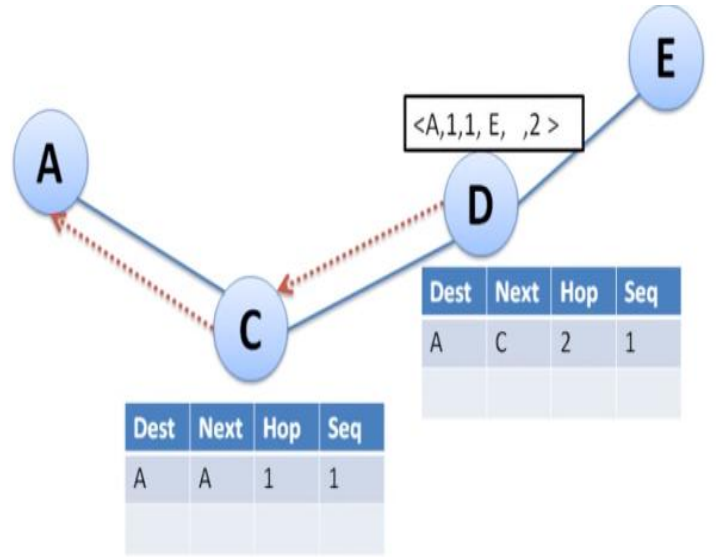

At this point ,as well as packet reachs ' $\mathrm{D}$ ' it will make an entry in its table like above i.e. in order to reach destination A we need to go to 'next-c'and its hop count is ' 2 '.

A wants to send message for $\mathrm{E}$ so route request is broadcasted.

Step 1: Awill send Route Request packet to all its neighbour.

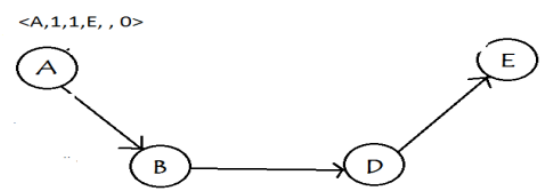

Step 2: A broadcast the packet to $\mathrm{C}$. but $\mathrm{C}$ does't have any info about ' $E$ '. $C$ broadcast the packet again . Before broadcasting that packet, $\mathrm{C}$ update the hopcount. Hop count is actually the distance of that particular node which is handling request from sender.

All the node maintain the following routing table.

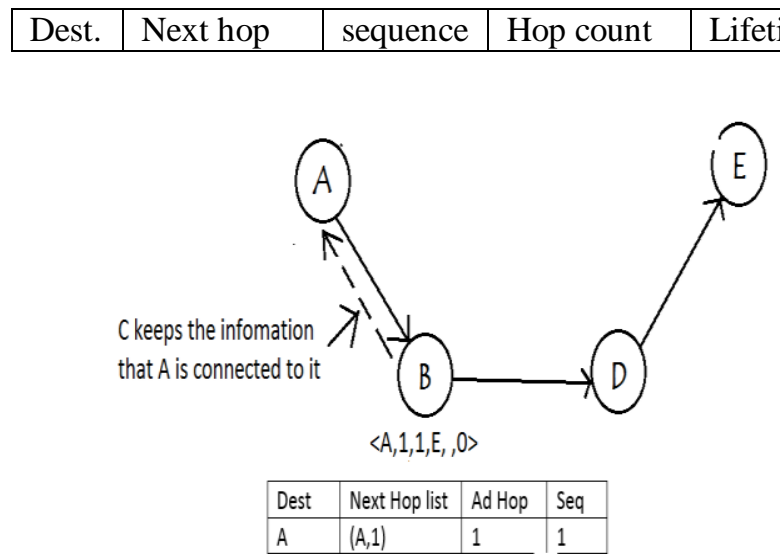

\section{Step 4:}

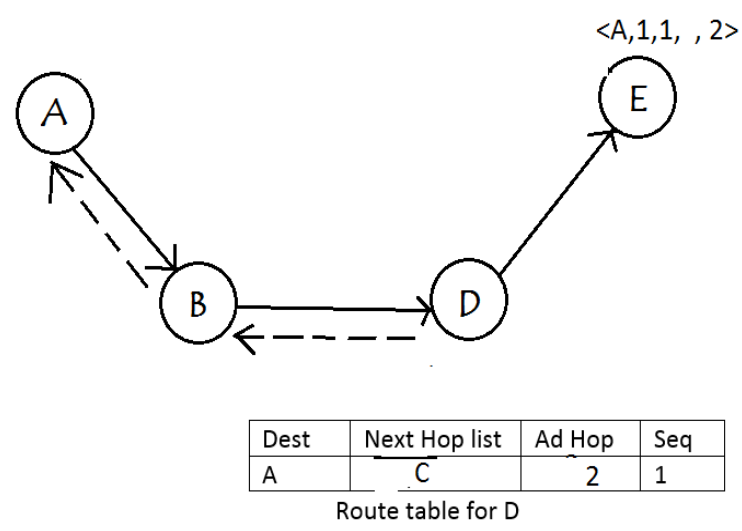

Step 5:

As soon as packet reaches the ' $E$ ' destination $E$ start preparing route reply packet.

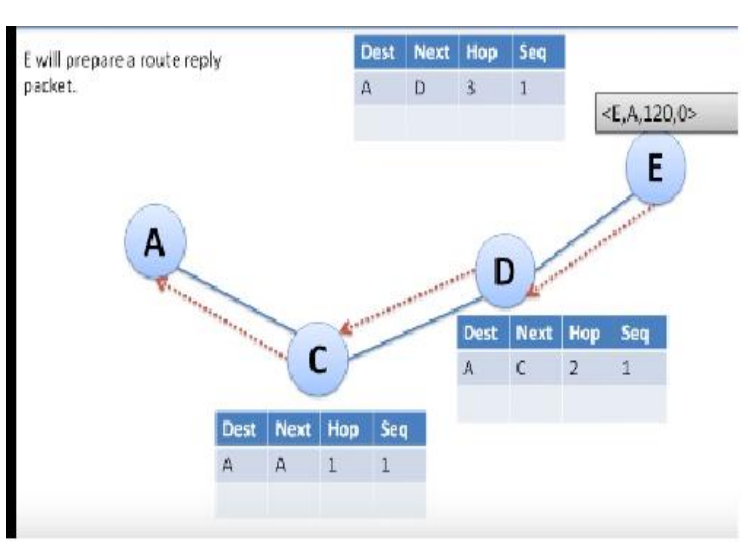


Step 6:

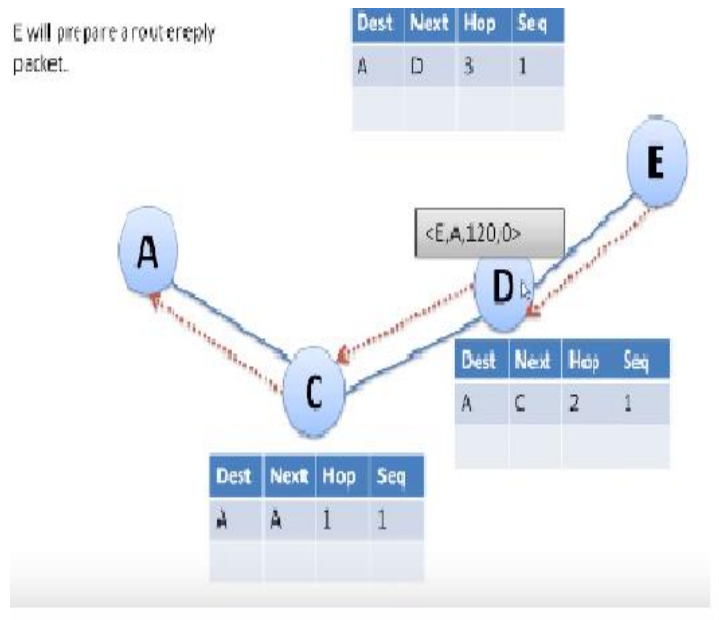

Step 7: Now D increase hop count from 0 to 1 and send the packet ahead.

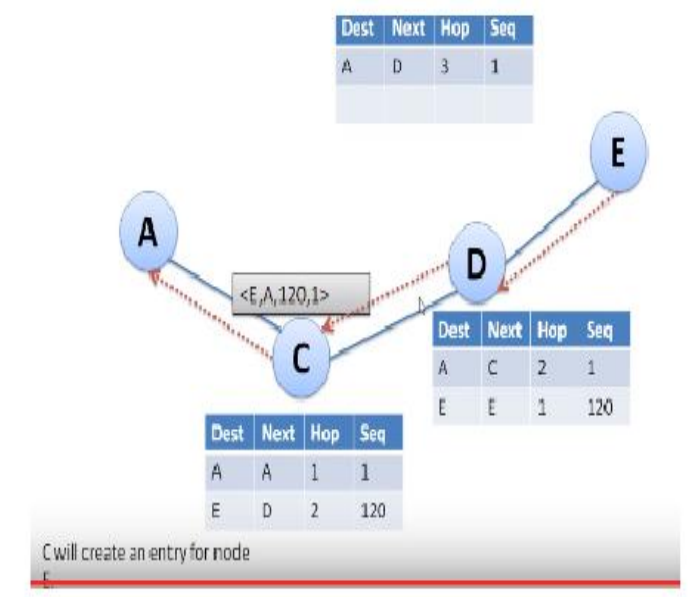

Step 8: Again C increase the hopcount from 1 to 2 and send the packet to $\mathrm{A}$.

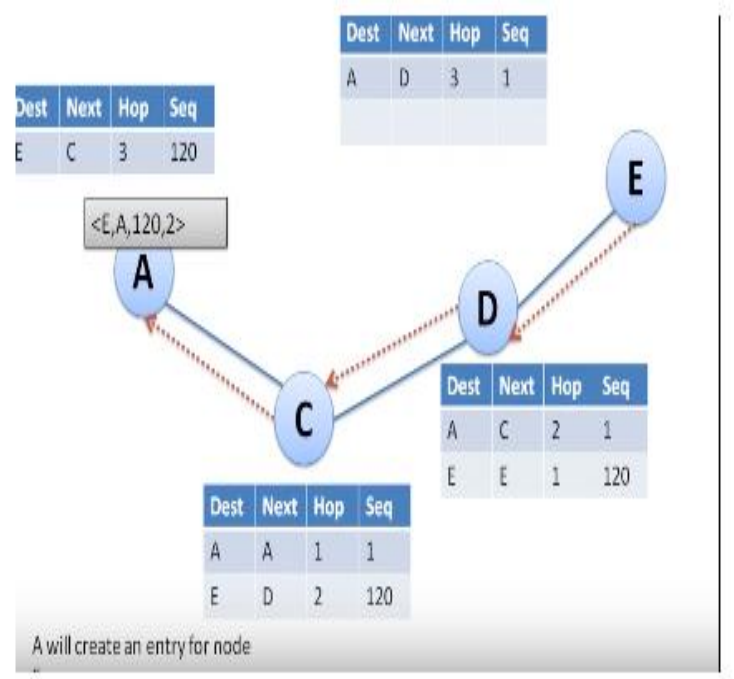

\section{CONCULSION}

In summary,we have presented a distance vector algorithm that is suitable for use with ad_hoc networksAODV avoids problems with previous proposals (no tably DSDV), and has the following features,

- $\quad$ Nodes store only the routes that are needed

- $\quad$ Need for broadcast is minimized

- Reduces memory requirements and needless duplications

- $\quad$ Quick response to link breakage in active routes

- Loop_free routes maintained by use of destination sequence numbers

- $\quad$ Scalable to large populations of nodes

We conclude that, within the limits imposed by worst_case route establishment latency as determined by the network diameter, AODV is an excellent choice for ad_hoc network establishment. It will be useful in applications for emergency services, conferencing battlefield communications, and community_based networking.We look forward to further development of the protocol for quality of service, intermediate route rebuilding , and various interconnection topologies with Fixed networks and the Internet.

\section{RESULT}

[RREQmat,np]=NL_R_AODVRouteRequestIn(g,Source, Dest,TTL)//Initialize the route request packet...RREQmat=request packet storage matrix,np=number of packets in the matrix $\mathrm{np}=2$.

RREQmat =

1. 6. 0.0 . $0.0 .0 . \quad 2 . \quad 390.23277$

1. 8 . 0 . 0.0 . 0.0 . 2. 118.37568

[RREQmat,np]=NL_R_AODVRouteRequestIt $(\mathrm{g}$, Source,D est,RREQmat,np)//Iterate the route request $\mathrm{np}=4$.

RREQmat =

1. 6. 7. $0.0 .0 . \quad 0.4 .443 .75608$

$\begin{array}{lllllllll}1 . & 8 . & 0 . & 0 . & 0 . & 0 . & 2 . & 118.37568\end{array}$

1. 6. 3. 0.0 .0 .0 .3 .826 .02961

1. 6. 5. $0.0 . \quad$ 1. 3. 650.79821

\section{REFERENCES}

[1] AODV, "Ad-hoc On-demand Distance Vector Routing", RFC 3561.

[2] C.E. Perkins, and E.M. Royer, Ad-hoc On-demand Distance Vector Routing, in: Proceedings of the 2th IEEE Workshop on Mobile Computing Systems and Applications, New Orleans, LA, February 1999, pp.90-100.

[3] A New Algorithm AODV Routing Protocol in Mobile ADHOC Networks Ali Khosrozadeh1, Abolfazle Akbari2, Maryam Bagheri and Neda Beikmahdavi Department of Computer Engineering Ayatollah Amoli Branch Islamic Azad University,Amol,Iran 